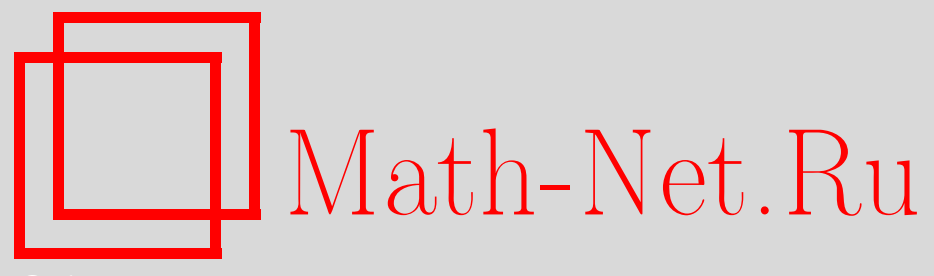

Н. А. Тюрин, Псевдоторические структуры и лагранжева сфера в многообразии флагов $F^{3}$, Матем. заметки, 2014, том 96, выпуск 3, 476-479

DOI: https://doi.org/10.4213/mzm10519

Использование Общероссийского математического портала Math-Net.Ru подразумевает, что вы прочитали и согласны с пользовательским соглашением http://www.mathnet.ru/rus/agreement

Параметры загрузки:

IP: 107.22 .136 .117

26 апреля 2023 г., 16:48:56

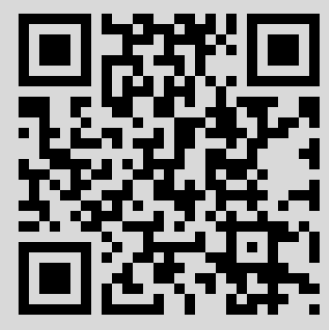




\section{Псевдоторические структуры и лагранжева сфера в многообразии флагов $F^{3}$}

\section{Н. А. Тюрин}

1. Псевдоторическая структура. Кратко напомним конструкцию псевдоторической структуры ранга 1 на пространстве флагов $F^{3}$ (детали см. в [1], общее определение псевдоторических структур может быть найдено в [2]).

Реализуем $F^{3}$ как гиперповерхность $\left\{\mathscr{U} \in \mathbb{C P}_{x}^{2} \times \mathbb{C P}_{y}^{2}\right\}$, задаваемую уравнением

$$
\sum_{i=0}^{2} x_{i} y_{i}=0
$$

на прямом произведении проективных плоскостей с однородными координатами $\left[x_{0}: x_{1}: x_{2}\right]$ и $\left[y_{0}: y_{1}: y_{2}\right]$ на $\mathbb{C P}_{x}^{2}$ и $\mathbb{C P}_{y}^{2}$ соответственно. Вещественная функция

$$
F\left(\lambda_{i} ; \mu_{j}\right)=\frac{\sum_{i=0}^{2} \lambda_{i}\left|x_{i}\right|^{2}}{\sum_{i=0}^{2}\left|x_{i}\right|^{2}}+\frac{\sum_{j=0}^{2} \mu_{j}\left|y_{j}\right|^{2}}{\sum_{j=0}^{2}\left|y_{j}\right|^{2}}
$$

сохраняется гамильтоновым действием $\mathscr{U}$, если и только если

$$
\lambda_{0}+\mu_{0}=\lambda_{1}+\mu_{1}=\lambda_{2}+\mu_{2}
$$

например, можно взять $\lambda_{i}=-\mu_{i}$. Таким образом, имеем в точности две вещественные функции Морса такого типа, которые алгебраически независимы почти всюду на $\mathscr{U}$ и коммутируют относительно скобки Пуассона, индуцируемой прямым произведением стандартных симплектических форм с прямых сомножителей (детали см. в [1]).

Выберем

$$
F_{1}=F(0,1,-2 ; 0,-1,2), \quad F_{2}=F(1,0,-2 ;-1,0,2) ;
$$

эти функции имеют попарно несовпадающие критические значения на $\mathscr{U}$ (но не на объемлющем пространстве). Рассмотрим отображение

$$
\psi: \mathbb{C P}_{x}^{2} \times \mathbb{C P}_{y}^{2} \rightarrow \mathbb{C P}_{w}^{2},
$$

задаваемое формулой $w_{i}=x_{i} y_{i}$; оно не определено в точности на шести прямых, соединяющих критические точки $F_{i}$ на $\mathscr{U}$. Ограничим это отображение на $\mathscr{U}$ и обозначим той же буквой $\psi$ так, что

$$
\psi: \mathscr{U} \backslash(6 \text { прямых }) \rightarrow L=\left\{\sum_{i=0}^{2} w_{i}=0\right\} \subset \mathbb{C P}_{w}^{2}
$$

Эти 6 прямых являются базисным множеством пучка дивизоров

$$
D_{\alpha, \beta}=\overline{\psi^{-1}([\alpha: \beta])}
$$

где в качестве однородных координат $[\alpha: \beta]$ можно взять $\alpha=w_{1}, \beta=w_{2}$ на $L$, причем (см. [1]) каждый $D_{\alpha, \beta}$ сохраняется гамильтоновым действем каждой $F_{i}$, т.е. ограничение

Работа выполнена при поддержке Лаборатории алгебраической геометрии НИУ-ВШЭ, и гранта правительства РФ аг. 11.G34.31.0023.

DOI: $10.4213 / \mathrm{mzm} 10519$

(C) Н. А. Тюрин, 2014 
$\left(\left.F_{1}\right|_{D_{\alpha, \beta}},\left.F_{2}\right|_{D_{\alpha, \beta}}\right)$ дает вполне интегрируемую систему на $D_{\alpha, \beta}$. Тип торического многообразия $D_{\alpha, \beta}$ определеяется выпуклым шестиугольником $P \subset \mathbb{R}^{2}$, откуда видно, что гладкий компактифицированный слой над точкой $[\alpha, \beta] \in L$ есть поверхность дель Пеццо $\mathbb{C P}_{3}^{2}$; негладкие слои надлежат в точности трем точкам в $L$, а именно

$$
p_{1}=[1:-1: 0], \quad p_{2}=[1: 0:-1], \quad p_{3}=[0: 1:-1],
$$

и являются объединениями пар поверхностей дель Пеццо типа $\mathbb{C P}_{1}^{2}$ (см. [1]).

Набор данных $\left(F_{i}, \psi\right)$ есть пседоторическая структура на многообразии флагов, используя которую, в [1] было построено лагранжево слоение с общим слоем гладкий лагранжев тор.

\section{2. Лагранжева сфера в $F^{3}$.}

ПрЕДЛОЖЕНИЕ 1. Въбор гладкого пути с концами в $p_{i}$ и $p_{j}$ на проективной прямой $L$, не проходящего через $p_{k}$, задает лагранжево вложение $S^{3}$ в $F^{3}$.

Здесь $(i, j, k)$ - произвольная перестановка $(1,2,3)$, а точки $p_{i}, p_{j}, p_{k}$ определены в (1).

В самом деле, пусть гладкий путь $\gamma:[0,1] \rightarrow L$ начинается в $p_{1}$ и кончается в $p_{2}$. Зафиксируем значения $F_{1}=F_{2}=0$. Тогда над каждой внутренней точкой $p \in \gamma \subset L, p \neq p_{i}$, это условие высекает на компактифицированном слое $D_{p}=\overline{\psi^{-1}(p)}$ совместное множество уровня $\left\{F_{1}=0, F_{2}=0\right\}$, являющееся гладким тором $T_{p}^{2} \subset D_{p}$. Над граничными точками $p_{1}, p_{2}$ на компактифицированных слоях $D_{p_{1}}, D_{p_{2}}$ зафиксированные значения $F_{1}, F_{2}$ соответствуют прообразам пересечения трех главных диагоналей в шестиугольнике $P$. Это означает, что условие $\left\{F_{1}=0, F_{2}=0\right\}$ высекает окружности на $D_{p_{1}}, D_{p_{2}}$, лежащие на прямых пересечения пар поверхностей дель Пеццо, составляющих особые компактифицированные слои. Соберем по всем слоям $D_{p}, p \in \gamma$, множества уровней $\left\{F_{1}=0, F_{2}=0\right\}$ :

$$
S=\bigcup_{p \in \gamma} D_{p} \cap\left\{F_{1}=0, F_{2}=0\right\} ;
$$

подмногообразие $S \subset F^{3}$ лагранжево по построению, как и торы из [1], так что нам необходимо показать, что $S$ является трехмерной сферой. Мы сделаем это в два шага: сначала построим похожую псевдоторическую структуру на $\mathbb{C P}^{3}$ и опишем в этих терминах лагранжево $\mathbb{R P}^{3}$, а затем в качестве второго шага покажем, что наше $S$ двулистно накрывает это последнее.

Рассмотрим $\mathbb{C P}^{3}$ с однородными координатами $\left[z_{0}: z_{1}: z_{2}: z_{3}\right]$ и псведоторической структурой $\left(G_{1}, G_{2}, \phi\right)$, построенной в [2], где $\phi: \mathbb{C P}^{3} \backslash B \rightarrow \mathbb{C P}_{\alpha, \beta}^{1}$ задается как

$$
\overline{\phi^{-1}(p)}=\left\{\alpha z_{0} z_{1}=\beta z_{2} z_{3}\right\}, \quad p \mapsto[\alpha, \beta] \in \mathbb{C P}_{\alpha, \beta}^{1},
$$

где базисное множество $B \subset \mathbb{C P}^{3}$ состоит из 4 прямых, а функции $G_{1}, G_{2}$ имеют вид

$$
G_{1}=\frac{\left|z_{0}\right|^{2}-\left|z_{1}\right|^{2}+2\left|z_{2}\right|^{2}-2\left|z_{3}\right|^{2}}{\sum_{i=0}^{3}\left|z_{i}\right|^{2}}, \quad G_{2}=\frac{2\left|z_{0}\right|^{2}-2\left|z_{1}\right|^{2}+\left|z_{2}\right|^{2}-\left|z_{3}\right|^{2}}{\sum_{i=0}^{3}\left|z_{i}\right|^{2}}
$$

особые слои надлежат $[1: 0]$ и $[0: 1]$, каждый из них есть объединение двух плоскостей, пересекающихся по прямой. Зафиксируем $G_{1}=G_{2}=0$ и возьмем отрезок $\alpha=t, \beta=t-1$, $t \in[0 ; 1]$, на проективной прямой $\mathbb{C P}_{\alpha, \beta}^{1}$. На кажом слое

$$
D_{p_{t}}^{\prime}=\overline{\phi^{-1}\left(p_{t}\right)}, \quad t \neq 0,1,
$$

условия $\left\{G_{1}=G_{2}=0\right\}$ высекают лагранжев тор на $D_{p_{t}}^{\prime}$, а над концами $p_{0}, p_{1}$ мы снова имеем окружности. Получаем

$$
S_{0}=\bigcup_{t \in[0 ; 1]} D_{p_{t}}^{\prime} \cap\left\{G_{1}=0, G_{2}=0\right\}
$$


покажем явно, что это лагранжево $\mathbb{R} \mathbb{P}^{3}$ в $\mathbb{C P}^{3}$. В самом деле, $S_{0}$ лагранжево по построению: рассуждение в точности такое же, как в [1], [2] для торов. Далее, покажем что $S_{0}$ есть $\mathbb{R P}^{3}$. Выберем другие однородные координаты $\left[x_{0}: x_{1}: x_{2}: x_{3}\right]$ на $\mathbb{C P}^{3} ;$ в них стандартное $\mathbb{R P}^{3}$ задается условием $\left\{x_{i} \in \mathbb{R}\right\}$. Пусть $z$-координаты и $x$-координаты связаны следующим проективным преобразованием:

$$
\left[x_{0}: x_{1}: x_{2}: x_{3}\right] \mapsto\left[z_{0}=x_{0}+\imath x_{1}: z_{1}=\imath x_{0}+x_{1}: z_{2}=x_{2}+\imath x_{3}: z_{3}=\imath x_{2}+x_{3}\right] ;
$$

тогда условие $\left\{x_{i} \in \mathbb{R}\right\}$ преобразуется в условия

$$
\left|z_{0}\right|=\left|z_{1}\right|, \quad\left|z_{2}\right|=\left|z_{3}\right|, \quad t z_{0} z_{1}=(t-1) z_{2} z_{3}
$$

для некоторых $t$, лежащих на отрезке $[0 ; 1]$. То есть мы получаем, что $S_{0}$ не только изоморфна $\mathbb{R P}^{3}$, но еще и гамильтоново изотопна стандартному вещественному проективному пространству.

Далее, покажем, что $S$ двулистно накрывает $S_{0}$. Пусть путь $\gamma \subset L$ соединяет точки $[1$ : $-1: 0]$ и $[1: 0:-1]$. Вырежем из баз псевдоторических структур $L$ и $\mathbb{C P}_{\alpha, \beta}^{1}$ по точке: из $L$ точку с координатами $[0: 1:-1]$, а из $\mathbb{C P}_{\alpha, \beta}^{1}$ точку $[1:-1]$. Заметим, что это вырезание не задевает наши лагранжевы подмногообразия $S$ и $S_{0}$. Посмотрим, как будут выглядеть $S$ и $S_{0}$ при аффинизации $\mathscr{U}$ и $\mathbb{C P}^{3}$, возникающей после этого вырезания.

Множество

$$
K_{0}=\mathbb{C P}^{2} \backslash Q_{1,-1}
$$

- проективное пространство без квадрики $Q=\left\{z_{0} z_{1}+z_{2} z_{3}=0\right\}$ - двулистно накрывается гиперповерхностью $\left\{Z_{0} Z_{1}+Z_{2} Z_{3}=1\right\} \in \mathbb{C}^{4}$ без ветвлений. В то же время $\mathscr{U} \backslash D_{1,-1}$ может быть представленно аффинной моделью в $\mathbb{C}^{2} \times \mathbb{C}^{2}$ если, воспользовавшись описанием $D_{1,-1}$ как $x_{0} y_{0}=0$ в $\mathbb{C P}_{x}^{2} \times \mathbb{C P}_{y}^{2}$, мы возьмем в каждом прямом слагаемом аффинные карты $x_{0} \neq 0, y_{0} \neq 0$. Тогда нетрудно видеть, что в аффинных координатах $\mathbb{C}^{2} \times \mathbb{C}^{2}$ нужная нам аффинная часть $\mathscr{U}$ задается уравнением $K=\left\{X_{1} Y_{1}+X_{2} Y_{2}=1\right\}$. Таким образом, после выбрасываения слоя над одной и той же точкой базы псевдоторических структур мы получаем двулистное накрытие $K \rightarrow K_{0}$.

Далее, оба аффинных многообразия расслоены над общей базой $\mathbb{C P}_{\alpha, \beta}^{1}=L$ с выколотой точкой, и нетрудно видеть, что слои $\psi$ и $\phi$ совпадают. Условия зануления функций $F_{1}, F_{2}$, $G_{1}, G_{2}$ в точности соответствуют попарному равенству норм:

$$
\begin{array}{ccl}
F_{1}=F_{2}=0 & \Longleftrightarrow \quad\left|X_{1}\right|=\left|Y_{1}\right|, \quad\left|X_{2}\right|=\left|Y_{2}\right|, \\
G_{1}=G_{2}=0 & \Longleftrightarrow \quad\left|Z_{0}\right|=\left|Z_{1}\right|, \quad\left|Z_{2}=\right| Z_{3} \mid .
\end{array}
$$

В результате получаем, что условия, определяющие $S$ и $S_{0}$, совпадают по модулю двулистности накрытия $K \rightarrow K_{0}$, откуда следует необходимое нам утверждение.

Таким образом, $S$ является лагранжевой сферой.

В заключение приведем несколько естественных замечаний.

При вариациях гладкого пути $\gamma \in L$ с фиксированными концами мы получаем лагранжевы деформации $S$, а поскольку периоды лагранжевой сферы при непрерывных деформациях не могут изменяться, эти деформации являются гамильтоновыми изотопиями. Отсюда следует, что все лагранжевы сферы, получаемые нашей конструкцией при фиксированных концах $p_{i}, p_{j}$, гамильтоново изотопны. Однако совсем не очевидна гамильтонова изотопность лагранжевых сфер, получающихся при выборе разных пар концов. Нетрудно видеть, что такие сферы симплектоморфны, но очень правдоподобной выглядит гиптеза о том, что они гамильтоново неэквивалентны. Препятствия очень похожи на те, которые возникают при деформациях чекановских торов. Более того, естественно встает вопрос о том, является ли лагранжева сфера, доставляемая нашей конструкцией, известной лагранжевой сфере, возникающей из исследований системы Гельфанда-Цейтлина на многообразии флагов.

Представленная выше конструкция возникла как некоторое обобщение метода построения лагранжевых сфер из исчезающих циклов, индуцируемых пучками Лефшеца (см. [3]). 
Мы используем пучки, не являющиеся в строгом смысле пучками Лефшеца, поскольку наши отображения $\psi$ и $\phi$ имеют целые компоненты особенностей, а не только изолированные особенности. Однако, как мы видим, такие пучки тоже оказываются полезны в исследованиях лагранжевой геометрии алгебраических многообразий.

\section{СПИСОК ЦИТИРОВАННОЙ ЛИТЕРАТУРЫ}

[1] Н. А. Тюрин, ТМФ, 167:2 (2011), 193-205. [2] Н. А. Тюрин, ТМФ, 162:3 (2010), 307-333. [3] S. K. Donaldson, Mathematics: Frontiers and Perspectives, Amer. Math. Soc., Providence, RI, 2000, 55-64.

\section{Н. А. Тюрин}

Поступило

Лаборатория теоретической 20.03.2014

физики им. Н. Н. Боголюбова,

ОИЯИ, г. Дубна Московской обл.

Национальный исследовательский университет

«Высшая школа экономики», г. Москва

E-mail: ntyurin@theor.jinr.ru 